\title{
The Role of Charge Transfer in Halogen Bonding
}

\author{
Brandon Inscoe, Hemali Rathnayake and Yirong Mo*
}

Department of Nanoscience, Joint School of Nanoscience and Nanoengineering, University of North Carolina at Greensboro, Greensboro, NC 27401, United States.

Table S1. Optimal geometries with both the regular DFT and BLW methods at the M06-2X$\mathrm{D} 3 / 6-311+\mathrm{G}(\mathrm{d}, \mathrm{p})(\mathrm{def} 2-\mathrm{SVP}$ for iodine) level of theory (file type, PDF)

\begin{tabular}{|c|c|c|}
\hline Structure & DFT Opt & BLW Opt \\
\hline $\mathrm{F}_{3} \mathrm{C}-\mathrm{Br}^{-\cdots \mathrm{F}^{-}}$ & $\begin{array}{llll}\text { C } & -2.1185014352 & -0.0007918809 & 0.0009596704 \\
\text { F } & -2.6525816453 & -1.2143807227 & 0.2709373313 \\
\text { F } & -2.6526922358 & 0.8394081944 & 0.9172753662 \\
\text { F } & -2.6535323743 & 0.3723441228 & -1.1845815931 \\
\text { BR } & -0.1647764998 & -0.0001647012 & -0.0001468081 \\
\text { F } & 2.0414221852 & 0.0004608611 & -0.0011661142\end{array}$ & $\begin{array}{llll}\text { C } & -2.1722644308 & -0.0007296868 & 0.0009443624 \\
\text { F } & -2.6884602151 & -1.2119699468 & 0.2736890539 \\
\text { F } & -2.6892564271 & 0.8403016851 & 0.9138395296 \\
\text { F } & -2.6903459499 & 0.3685220026 & -1.1836432729 \\
\text { BR } & -0.2759425212 & 0.0002251334 & -0.0003459594 \\
\text { F } & 2.3156075388 & 0.0005266860 & -0.0012058610\end{array}$ \\
\hline $\mathrm{Cl}_{3} \mathrm{C}-\mathrm{Br}^{-\cdots} \mathrm{F}^{-}$ & $\begin{array}{lccc}\text { C } & 1.4425742412 & -0.0018617300 & 0.0060991145 \\
\text { CL } & 2.0950210560 & -0.6504023945 & 1.5540690105 \\
\text { CL } & 2.1075864365 & -1.0160899407 & -1.3252779398 \\
\text { CL } & 2.1034436400 & 1.6603843541 & -0.2021322707 \\
\text { BR } & -0.5839912324 & -0.0012432267 & -0.0027566045 \\
\text { F } & -2.6823581413 & -0.0005000621 & -0.0118063100\end{array}$ & $\begin{array}{lccc}\text { C } & 1.5341993598 & -0.0023312310 & 0.0043722583 \\
\text { CL } & 2.1857715968 & -0.6526547318 & 1.5460344988 \\
\text { CL } & 2.1899006265 & -1.0129089407 & -1.3271829949 \\
\text { CL } & 2.1918520347 & 1.6556099085 & -0.2026912510 \\
\text { BR } & -0.3813827776 & 0.0001664644 & 0.0013708961 \\
\text { F } & -2.9380643455 & 0.0024050021 & -0.0037081453\end{array}$ \\
\hline $\mathrm{Br}_{3} \mathrm{C}-\mathrm{Br} \cdot \cdots \mathrm{F}^{-}$ & $\begin{array}{lccc}\text { C } & 0.2169717441 & 0.0059444418 & 0.0038329927 \\
\text { BR } & 0.9319288731 & -0.7013135402 & 1.7003659635 \\
\text { BR } & 0.9579465400 & -1.0952360904 & -1.4546134217 \\
\text { BR } & 0.9223652024 & 1.8341714591 & -0.2185462169 \\
\text { BR } & -1.8174865967 & -0.0125984866 & -0.0106326823 \\
\text { F } & -3.8928647629 & -0.0315407833 & -0.0254016353\end{array}$ & $\begin{array}{lccc}\text { C } & 0.9998261971 & 0.3474624605 & -0.7134589469 \\
\text { BR } & 0.4570686524 & 0.7577462897 & -2.5588226295 \\
\text { BR } & 1.8789556721 & 1.9504985415 & 0.0116825158 \\
\text { BR } & 2.3530838471 & -1.0771563862 & -0.8007629444 \\
\text { BR } & -0.4943172981 & -0.1726221116 & 0.3543129244 \\
\text { F } & -2.4929757519 & -0.8659709982 & 1.7781662494\end{array}$ \\
\hline $\mathbf{F}_{3} \mathrm{C}-\mathbf{I} \cdot \boldsymbol{F}^{-}$ & $\begin{array}{cccc}\text { C } & -2.4739473915 & 0.0008297943 & -0.0008775510 \\
\text { F } & -3.0236084181 & -1.2272989068 & 0.2007316121 \\
\text { F } & -3.0243367736 & 0.7886457071 & 0.9622325203 \\
\text { F } & -3.0253052751 & 0.4405210724 & -1.1643799634 \\
\text { I } & -0.2590277356 & 0.0012026005 & -0.0015130569 \\
\text { F } & 1.9634021003 & 0.0010923492 & -0.0008139693\end{array}$ & $\begin{array}{cccc}\text { C } & -2.5152177903 & 0.0006000435 & 0.0000499113 \\
\text { F } & -3.0432842065 & -1.2253138684 & 0.2049762586 \\
\text { F } & -3.0448320213 & 0.7898801320 & 0.9594187160 \\
\text { F } & -3.0464305208 & 0.4359362182 & -1.1626752637 \\
\text { I } & -0.3734054560 & 0.0017180513 & -0.0016417461 \\
\text { F } & 2.1803465014 & 0.0021720401 & -0.0047482842\end{array}$ \\
\hline $\mathrm{Cl}_{3} \mathrm{C}-\mathrm{I} \cdot \boldsymbol{F}^{-}$ & $\begin{array}{lccc}\text { C } & -2.4103074376 & 0.0012587297 & -0.0017777593 \\
\text { CL } & -3.0800404291 & 0.9519478993 & 1.3789241279 \\
\text { CL } & -3.0783204987 & 0.7228335240 & -1.5156652128 \\
\text { CL } & -3.0811186252 & -1.6694443463 & 0.1300350819\end{array}$ & $\begin{array}{lccc}\text { C } & -2.4381529831 & 0.0004007320 & -0.0017217631 \\
\text { CL } & -3.0987183279 & 0.9487162916 & 1.3748774633 \\
\text { CL } & -3.0962995467 & 0.7188673327 & -1.5121894580 \\
\text { CL } & -3.0972481592 & -1.6665855047 & 0.1304094554\end{array}$ \\
\hline
\end{tabular}




\begin{tabular}{|c|c|c|}
\hline & $\begin{array}{lrrr}\text { I } & -0.1310111155 & -0.0007146529 & -0.0000809261 \\
\text { F } & 2.0310007765 & -0.0029408046 & 0.0017838682\end{array}$ & $\begin{array}{lrrr}\text { I } & -0.2627917259 & 0.0006231234 & 0.0000566239 \\
\text { F } & 2.2434134132 & 0.0009183741 & 0.0017868585\end{array}$ \\
\hline $\mathbf{B r}_{3} \mathrm{C}-\mathbf{I} \cdot \cdot \mathbf{F}^{-}$ & $\begin{array}{lccc}\text { C } & -2.4000538244 & 0.0012994130 & -0.0010682923 \\
\text { BR } & -3.1284658734 & 1.1287289661 & 1.4481893304 \\
\text { BR } & -3.1266005442 & 0.6940984709 & -1.7022822421 \\
\text { BR } & -3.1303859702 & -1.8168959016 & 0.2496299083 \\
\text { I } & -0.1095158934 & -0.0008335219 & -0.0002279610 \\
\text { F } & 2.0372079149 & -0.0027656583 & 0.0002948034\end{array}$ & $\begin{array}{lccc}\text { C } & -2.4230393089 & 0.0012610874 & -0.0011138460 \\
\text { BR } & -3.1434836438 & 1.1251610332 & 1.4434256956 \\
\text { BR } & -3.1417260497 & 0.6917418817 & -1.6968408717 \\
\text { BR } & -3.1452373720 & -1.8112464098 & 0.2486091602 \\
\text { I } & -0.2498838194 & -0.0008199633 & -0.0000432058 \\
\text { F } & 2.2455560031 & -0.0024658610 & 0.0004986146\end{array}$ \\
\hline $\mathrm{F}_{3} \mathrm{C}-\mathrm{Br} \cdot{ }^{-\mathrm{Cl}^{-}}$ & $\begin{array}{llll}\text { C } & -2.2274796937 & -0.0009152349 & 0.0014067703 \\
\text { F } & -2.7372083960 & -1.2127524405 & 0.2745803944 \\
\text { F } & -2.7380041275 & 0.8401764341 & 0.9151680562 \\
\text { F } & -2.7403210343 & 0.3685580876 & -1.1833077035 \\
\text { BR } & -0.3022724537 & 0.0006039035 & -0.0012356975 \\
\text { CL } & 2.6446236999 & 0.0012051237 & -0.0033339673\end{array}$ & $\begin{array}{llll}\text { C } & -2.2798047931 & -0.0006631332 & 0.0010906347 \\
\text { F } & -2.7798531883 & -1.2126173183 & 0.2688405828 \\
\text { F } & -2.7803405181 & 0.8362661494 & 0.9174699944 \\
\text { F } & -2.7823640447 & 0.3736048076 & -1.1813095198 \\
\text { BR } & -0.3802726411 & 0.0003982064 & -0.0009556274 \\
\text { CL } & 2.9019731801 & -0.0001128384 & -0.0018582123\end{array}$ \\
\hline $\mathrm{Cl}_{3} \mathrm{C}$ & $\begin{array}{lccc}\text { C } & 1.5322260344 & -0.0017842655 & 0.0068968381 \\
\text { CL } & 2.1651385085 & -0.6494941322 & 1.5533367537 \\
\text { CL } & 2.1791511776 & -1.0145437539 & -1.3226021204 \\
\text { CL } & 2.1741008896 & 1.6587062741 & -0.2007487933 \\
\text { BR } & -0.4543373060 & -0.0014892705 & -0.0027783925 \\
\text { CL } & -3.2140033040 & -0.0011078519 & -0.0159092855\end{array}$ & $\begin{array}{lccc}\text { C } & 1.5946992787 & -0.0017653640 & 0.0088782985 \\
\text { CL } & 2.2198468325 & -0.6482849173 & 1.5543717765 \\
\text { CL } & 2.2398062135 & -1.0133083838 & -1.3169526368 \\
\text { CL } & 2.2325183178 & 1.6561790241 & -0.1972150056 \\
\text { BR } & -0.3235670378 & -0.0015266035 & -0.0043703537 \\
\text { CL } & -3.5810276049 & -0.0010067553 & -0.0265170790\end{array}$ \\
\hline $\mathrm{Br}_{3} \mathrm{C}$ & $\begin{array}{lccc}\text { C } & 0.3014665632 & 0.0072311148 & 0.0049576981 \\
\text { BR } & 0.9983008125 & -0.6995628482 & 1.7003767186 \\
\text { BR } & 1.0259919973 & -1.0928559700 & -1.4522368111 \\
\text { BR } & 0.9885896742 & 1.8340777002 & -0.2169636832 \\
\text { BR } & -1.6918849043 & -0.0118158526 & -0.0102955707 \\
\text { CL } & -4.4036031429 & -0.0376471439 & -0.0308333517\end{array}$ & $\begin{array}{lccc}\text { C } & 0.3660013345 & 0.0087326639 & 0.0064234897 \\
\text { BR } & 1.0569639364 & -0.6956566301 & 1.6992014839 \\
\text { BR } & 1.0892569931 & -1.0883882571 & -1.4465277962 \\
\text { BR } & 1.0479668088 & 1.8323319150 & -0.2141402337 \\
\text { BR } & -1.5452051177 & -0.0111566498 & -0.0107931601 \\
\text { CL } & -4.7961229550 & -0.0464360415 & -0.0391587837\end{array}$ \\
\hline $\mathbf{F}_{3} \mathbf{C}$ & $\begin{array}{lccc}\mathrm{C} & -2.5661949114 & 0.0006772690 & -0.0002300927 \\
\mathrm{~F} & -3.0944647311 & -1.2267413859 & 0.1985532548 \\
\mathrm{~F} & -3.0937911340 & 0.7859272395 & 0.9642350692 \\
\mathrm{~F} & -3.0963317478 & 0.4428156347 & -1.1615534099 \\
\mathrm{I} & -0.3784021430 & 0.0009837620 & -0.0024583780 \\
\mathrm{CL} & 2.4863611737 & 0.0013300973 & -0.0031668515\end{array}$ & $\begin{array}{lrrr}\text { C } & -2.6271909872 & 0.0006515112 & -0.0008524545 \\
\text { F } & -3.1378091533 & -1.2246684029 & 0.1997609582 \\
\text { F } & -3.1380075647 & 0.7871671116 & 0.9598187096 \\
\text { F } & -3.1377367838 & 0.4395128535 & -1.1623779281 \\
\text { I } & -0.4938735782 & 0.0006403516 & -0.0004811583 \\
\text { CL } & 2.7917945736 & 0.0016891917 & -0.0004885352\end{array}$ \\
\hline $\mathrm{Cl}_{3} \mathrm{C}-\mathrm{I} \cdot \cdot \mathrm{Cl}^{-}$ & $\begin{array}{lccc}\text { C } & -2.5088309236 & 0.0012864750 & -0.0016923167 \\
\text { CL } & -3.1596948808 & 0.9516405306 & 1.3775305990 \\
C L & -3.1575088429 & 0.7222824998 & -1.5148936457 \\
\text { CL } & -3.1613644863 & -1.6681210977 & 0.1299765988 \\
\text { I } & -0.2476046881 & -0.0008202042 & 0.0002376473 \\
C L & 2.4852064921 & -0.0033278542 & 0.0020602970\end{array}$ & $\begin{array}{lccc}C & -2.5668873633 & 0.0010633596 & 0.0007298068 \\
\text { CL } & -3.2041525431 & 0.9482069224 & 1.3786091508 \\
\text { CL } & -3.2091337048 & 0.7203537235 & -1.5066490237 \\
\text { CL } & -3.2068458196 & -1.6649265826 & 0.1333897782 \\
\text { I } & -0.4052654708 & 0.0004664820 & -0.0031422120 \\
C L & 2.8424875720 & -0.0022235556 & -0.0097183204\end{array}$ \\
\hline $\mathrm{Br}_{3} \mathrm{C}-\mathrm{I} \cdots \mathrm{Cl}^{-}$ & $\begin{array}{lccc}\text { C } & -2.4788382954 & 0.0016275511 & -0.0013737893 \\
\text { BR } & -3.1886847318 & 1.1285069039 & 1.4474130793 \\
\text { BR } & -3.1873150978 & 0.6950288781 & -1.7016786001 \\
\text { BR } & -3.1920783916 & -1.8153368895 & 0.2483912381 \\
\text { I } & -0.2062962236 & -0.0013726646 & 0.0000119534 \\
\text { CL } & 2.4953985497 & -0.0048220109 & 0.0017716653\end{array}$ & $\begin{array}{lccc}\text { C } & -2.5348453329 & 0.0015484791 & -0.0016024929 \\
\text { BR } & -3.2362090903 & 1.1259426284 & 1.4417242102 \\
\text { BR } & -3.2334677387 & 0.6912941545 & -1.6973006956 \\
\text { BR } & -3.2385469726 & -1.8097918070 & 0.2485557526 \\
\text { I } & -0.3770688902 & -0.0011472007 & 0.0004465468 \\
\text { CL } & 2.8623238341 & -0.0042144861 & 0.0027122256\end{array}$ \\
\hline $\mathrm{F}_{3} \mathrm{C}-\mathrm{Br} \cdot \cdot \mathrm{Br}^{-}$ & $\begin{array}{llll}\mathrm{C} & -2.3923426627 & -0.0009502260 & 0.0012484414 \\
\mathrm{~F} & -2.8984473752 & -1.2130648278 & 0.2727178905\end{array}$ & $\begin{array}{llll}\mathrm{C} & -2.3987621149 & -0.0006810769 & 0.0016993684 \\
\mathrm{~F} & -2.8948123615 & -1.2110996052 & 0.2771992527\end{array}$ \\
\hline
\end{tabular}




\begin{tabular}{|c|c|c|}
\hline & $\begin{array}{lccc}\text { F } & -2.8995010907 & 0.8391827218 & 0.9155846901 \\
\text { F } & -2.9009484211 & 0.3699037795 & -1.1831967425 \\
\text { BR } & -0.4666328134 & 0.0004659980 & -0.0004694604 \\
\text { BR } & 2.6572103579 & 0.0013384279 & -0.0026069665\end{array}$ & $\begin{array}{lccc}\text { F } & -2.8957987280 & 0.8416767568 & 0.9130109293 \\
\text { F } & -2.8985072635 & 0.3659968145 & -1.1827535865 \\
\text { BR } & -0.4979231208 & 0.0009110701 & -0.0013539045 \\
\text { BR } & 2.9851415835 & 0.0000719143 & -0.0045242069\end{array}$ \\
\hline $\mathrm{Cl}_{3} \mathrm{C}-\mathrm{Br} \cdot \cdot \mathrm{Br}^{-}$ & $\begin{array}{lccc}\text { C } & 1.6465408988 & -0.0016476745 & 0.0073634534 \\
\text { CL } & 2.2743266512 & -0.6499682813 & 1.5537923803 \\
\text { CL } & 2.2888602581 & -1.0142552585 & -1.3221177102 \\
\text { CL } & 2.2844508735 & 1.6584108954 & -0.1997534263 \\
\text { BR } & -0.3415156984 & -0.0009363944 & -0.0028571692 \\
\text { BR } & -3.2703869831 & -0.0013162866 & -0.0182325278\end{array}$ & $\begin{array}{lccc}\text { C } & 1.5473582357 & -0.0016777558 & 0.0073904699 \\
\text { CL } & 2.1722595752 & -0.6486061316 & 1.5512918985 \\
\text { CL } & 2.1854715295 & -1.0129688636 & -1.3202962105 \\
\text { CL } & 2.1807090641 & 1.6562375515 & -0.2000266330 \\
\text { BR } & -0.3722375142 & -0.0013995290 & -0.0015817865 \\
\text { BR } & -3.8312848902 & -0.0012982712 & -0.0185827383\end{array}$ \\
\hline $\mathrm{Br}_{3} \mathrm{C}$ & $\begin{array}{lccc}\text { C } & 0.3985213931 & 0.0073193383 & 0.0051968605 \\
\text { BR } & 1.0906048680 & -0.7001911802 & 1.7005605997 \\
\text { BR } & 1.1181410387 & -1.0930598012 & -1.4524959876 \\
\text { BR } & 1.0819237613 & 1.8339976716 & -0.2164624380 \\
\text { BR } & -1.5967550652 & -0.0110617061 & -0.0100406260 \\
\text { BR } & -4.4735749958 & -0.0375773222 & -0.0317534086\end{array}$ & $\begin{array}{lccc}\text { C } & 0.3688393206 & 0.0084735954 & 0.0061481867 \\
\text { BR } & 1.0566962695 & -0.6969708656 & 1.6980014124 \\
\text { BR } & 1.0857172576 & -1.0887626963 & -1.4479150114 \\
\text { BR } & 1.0471073736 & 1.8317773964 & -0.2146542871 \\
\text { BR } & -1.5438479866 & -0.0104318545 & -0.0091596272 \\
\text { BR } & -4.9956512346 & -0.0446585750 & -0.0374156734\end{array}$ \\
\hline $\mathrm{F}_{3} \mathrm{C}$ & $\begin{array}{cccc}\text { C } & -2.6637752994 & 0.0006089463 & 0.0004069007 \\
\text { F } & -3.1876652076 & -1.2268925216 & 0.1992235364 \\
\text { F } & -3.1873525414 & 0.7855667265 & 0.9650038816 \\
\text { F } & -3.1905378067 & 0.4427194903 & -1.1605411365 \\
\text { I } & -0.4766486822 & 0.0011537988 & -0.0025460743 \\
\text { BR } & 2.5631560437 & 0.0018361763 & -0.0061675160\end{array}$ & $\begin{array}{cccc}\text { C } & -2.6454313754 & 0.0005829465 & 0.0002924825 \\
\text { F } & -3.1519349191 & -1.2251178960 & 0.1990432518 \\
\text { F } & -3.1503968419 & 0.7844262600 & 0.9641173452 \\
\text { F } & -3.1546111892 & 0.4423469833 & -1.1589401792 \\
\text { I } & -0.5124854182 & 0.0004444603 & -0.0032290754 \\
\text { BR } & 2.9720362502 & 0.0023098625 & -0.0059042330\end{array}$ \\
\hline & $\begin{array}{lccc}\mathrm{C} & -2.5885559617 & 0.0011320787 & -0.0023965235 \\
\mathrm{CL} & -3.2360932020 & 0.9491798074 & 1.3781992622 \\
\mathrm{CL} & -3.2320437918 & 0.7245108659 & -1.51465666600 \\
\mathrm{CL} & -3.2362435439 & -1.6685886653 & 0.1263220456 \\
\mathrm{I} & -0.3244484910 & -0.0005816054 & 0.0008022811 \\
\text { BR } & 2.5675876607 & -0.0027121319 & 0.0049487803\end{array}$ & $\begin{array}{lccc}\text { C } & -2.6181431727 & 0.0015744971 & -0.0020519048 \\
\text { CL } & -3.2548597147 & 0.9507709058 & 1.3729081306 \\
\text { CL } & -3.2517953370 & 0.7190027701 & -1.5122037637 \\
\text { CL } & -3.2560164746 & -1.6635124046 & 0.1312199708 \\
\text { I } & -0.4573295862 & -0.0006215388 & 0.0004411791 \\
\text { BR } & 2.9883469556 & -0.0042738803 & 0.0029055676\end{array}$ \\
\hline $\mathbf{B r}_{3}$ & $\begin{array}{lccc}\text { C } & -2.5414558281 & 0.0016354603 & -0.0013752205 \\
\text { BR } & -3.2474222270 & 1.1284561109 & 1.4477752569 \\
\text { BR } & -3.2463646166 & 0.6946950137 & -1.7014768575 \\
\text { BR } & -3.2502690174 & -1.8159154048 & 0.2487456123 \\
\text { I } & -0.2653217024 & -0.0009610676 & -0.0002445638 \\
\text { BR } & 2.5930192009 & -0.0042783444 & 0.0011113193\end{array}$ & $\begin{array}{lccc}\text { C } & -2.6027386232 & 0.0017169949 & -0.0016491125 \\
\text { BR } & -3.2996508953 & 1.1246111630 & 1.4429740180 \\
\text { BR } & -3.2983241895 & 0.6928100475 & -1.6962670545 \\
\text { BR } & -3.3021155884 & -1.8100838388 & 0.2472938114 \\
\text { I } & -0.4460971682 & -0.0006102825 & -0.0003171659 \\
\text { BR } & 2.9911122741 & -0.0048123159 & 0.0025010503\end{array}$ \\
\hline $\mathrm{F}_{3} \mathrm{C}-\mathrm{Br} \boldsymbol{\cdots} \mathbf{I}^{-}$ & $\begin{array}{llll}\text { C } & -2.4232267815 & -0.0010755750 & 0.0013184256 \\
\text { F } & -2.9286386073 & -1.2133184231 & 0.2729065081 \\
\text { F } & -2.9299540348 & 0.8391733734 & 0.9156550635 \\
\text { F } & -2.9312948733 & 0.3696989126 & -1.1832474528 \\
\text { BR } & -0.4913641285 & 0.0005804135 & -0.0005668016 \\
\text { I } & 2.8038164202 & 0.0018171721 & -0.0027878903\end{array}$ & $\begin{array}{llll}\text { C } & -2.4706891660 & -0.0007237136 & 0.0012598971 \\
\text { F } & -2.9662769968 & -1.2124822054 & 0.2703568574 \\
\text { F } & -2.9661650311 & 0.8368702073 & 0.9173655359 \\
\text { F } & -2.9700653830 & 0.3726304946 & -1.1809482240 \\
\text { BR } & -0.5696410445 & 0.0003018946 & -0.0023471493 \\
\text { I } & 3.0421756162 & 0.0002791959 & -0.0024090645\end{array}$ \\
\hline $\mathrm{Cl}_{3} \mathrm{C}-\mathrm{Br}^{-\cdot \cdot \mathrm{I}^{-}}$ & $\begin{array}{lccc}\text { C } & 1.6481747635 & -0.0016371950 & 0.0073086501 \\
\text { CL } & 2.2720957064 & -0.6501861097 & 1.5543061999 \\
\text { CL } & 2.2869474034 & -1.0145657979 & -1.3225745913 \\
\text { CL } & 2.2820405724 & 1.6591064505 & -0.1999580870 \\
\text { BR } & -0.3569990142 & -0.0013167285 & -0.0029653534 \\
\text { I } & -3.4499834313 & -0.0011136193 & -0.0179218181\end{array}$ & $\begin{array}{lccc}\text { C } & 1.7032535729 & -0.0020005715 & 0.0078323002 \\
\text { CL } & 2.3250697784 & -0.6528675833 & 1.5507507581 \\
\text { CL } & 2.3401510600 & -1.0106458067 & -1.3216138155 \\
\text { CL } & 2.3367423777 & 1.6558049858 & -0.1952105343 \\
\text { BR } & -0.2167907554 & -0.0001806069 & -0.0024669656 \\
\text { I } & -3.8061500336 & 0.0001765827 & -0.0210967427\end{array}$ \\
\hline
\end{tabular}




\begin{tabular}{|c|c|c|}
\hline $\mathrm{Br}_{3} \mathrm{C}$ & $\begin{array}{lccc}\text { C } & 0.4006836180 & 0.0071882626 & 0.0050361166 \\
\text { BR } & 1.0891262399 & -0.7008956900 & 1.7017357381 \\
\text { BR } & 1.1171803763 & -1.0940886520 & -1.4536857946 \\
\text { BR } & 1.0812542865 & 1.8354708673 & -0.2166893043 \\
\text { BR } & -1.6152017237 & -0.0106107113 & -0.0099667590 \\
\text { I } & -4.6541817969 & -0.0376370765 & -0.0314249967\end{array}$ & $\begin{array}{lccc}\text { C } & 0.4584901093 & 0.0087801397 & 0.0062823758 \\
\text { BR } & 1.1445675641 & -0.6983447308 & 1.6976933109 \\
\text { BR } & 1.1716555824 & -1.0892742238 & -1.4482936679 \\
\text { BR } & 1.1382259361 & 1.8310326053 & -0.2143027500 \\
\text { BR } & -1.4549907021 & -0.0075147040 & -0.0082825327 \\
\text { I } & -5.0390874896 & -0.0452520863 & -0.0380917361\end{array}$ \\
\hline $\mathbf{F}_{3} \mathbf{C}$ & $\begin{array}{lrrr}\text { C } & -2.6647923422 & 0.0005849373 & 0.0004261696 \\
\text { F } & -3.1886042693 & -1.2270837312 & 0.1994930428 \\
\text { F } & -3.1876504606 & 0.7858269396 & 0.9653852766 \\
\text { F } & -3.1915040926 & 0.4426605620 & -1.1607189271 \\
\text { I } & -0.4629402845 & 0.0010672006 & -0.0029515750 \\
\text { I } & 2.7526679556 & 0.0019367083 & -0.0062543949\end{array}$ & $\begin{array}{cccc}\text { C } & -2.7187720688 & 0.0004962133 & -0.0002162864 \\
\text { F } & -3.2248468914 & -1.2251278138 & 0.1977792063 \\
\text { F } & -3.2241216113 & 0.7847093194 & 0.9626602481 \\
\text { F } & -3.2253341247 & 0.4422294100 & -1.1602703504 \\
\text { I } & -0.5858651685 & 0.0001884116 & -0.0012247074 \\
\text { I } & 3.0361163713 & 0.0024970761 & -0.0033485183\end{array}$ \\
\hline $\mathrm{Cl}_{3} \mathrm{C}$ & $\begin{array}{lccc}C & -2.5944266238 & 0.0010973347 & -0.0025810362 \\
C L & -3.2383497611 & 0.9495652789 & 1.3787647797 \\
C L & -3.2351753160 & 0.7246989118 & -1.5152865021 \\
C L & -3.2391235403 & -1.6692163052 & 0.1264857654 \\
I & -0.3071850983 & -0.0006075371 & 0.0005474742 \\
I & 2.7644630099 & -0.0025973337 & 0.0052886986\end{array}$ & $\begin{array}{llll}\text { C } & -2.6422864518 & 0.0016842291 & -0.0022500030 \\
\text { CL } & -3.2790007516 & 0.9472368630 & 1.3746399611 \\
\text { CL } & -3.2737066914 & 0.7234609411 & -1.5109418031 \\
\text { CL } & -3.2789516975 & -1.6637465112 & 0.1255076190 \\
\text { I } & -0.4812742144 & -0.0003871831 & 0.0013796580 \\
\text { I } & 3.1054224770 & -0.0053079895 & 0.0048837477\end{array}$ \\
\hline & $\begin{array}{lccc}\text { C } & -2.5478649025 & 0.0016998606 & -0.0013874112 \\
\text { BR } & -3.2503046945 & 1.1295009332 & 1.4491193910 \\
\text { BR } & -3.2493809717 & 0.6953389312 & -1.7031428050 \\
\text { BR } & -3.2526867049 & -1.8175433370 & 0.2490089662 \\
\text { I } & -0.2457538773 & -0.0009354726 & -0.0003024072 \\
\text { I } & 2.7881769604 & -0.0044291472 & 0.0012398128\end{array}$ & $\begin{array}{llll}\text { C } & -2.5939050196 & 0.0018071683 & -0.0017217079 \\
\text { BR } & -3.2890406044 & 1.1251099960 & 1.4429498924 \\
\text { BR } & -3.2887842905 & 0.6925783446 & -1.6963353327 \\
\text { BR } & -3.2916427007 & -1.8098903914 & 0.2478850508 \\
\text { I } & -0.4369598498 & -0.0004583196 & -0.0009102418 \\
\text { I } & 3.1425182744 & -0.0055150298 & 0.0026678858\end{array}$ \\
\hline $\mathrm{F}_{3} \mathrm{C}-\mathrm{Br} \cdots \mathrm{NMe}_{3}$ & $\begin{array}{lccc}\text { C } & -2.2413157261 & -0.0007206221 & 0.0010803551 \\
\text { F } & -2.7143965851 & -1.2130704936 & 0.2735813629 \\
\text { F } & -2.7157255333 & 0.8400406269 & 0.9153462904 \\
\text { F } & -2.7177712959 & 0.3695964203 & -1.1836844959 \\
\text { BR } & -0.3116115045 & 0.0006573227 & -0.0014886364 \\
\text { N } & 2.5001586396 & 0.0003726193 & -0.0015570235 \\
\mathrm{C} & 2.9302922671 & -0.4679406578 & 1.3059472596 \\
\mathrm{H} & 2.5370910059 & -1.4709189177 & 1.4838604575 \\
\mathrm{H} & 4.0290980451 & -0.5011534020 & 1.3939882472 \\
\mathrm{H} & 2.5397500223 & 0.1959177604 & 2.0799033864 \\
\mathrm{C} & 2.9415628163 & 1.3635382087 & -0.2482663373 \\
\mathrm{H} & 4.0410999189 & 1.4479639963 & -0.2597057678 \\
\mathrm{H} & 2.5569902894 & 1.7049128673 & -1.2114728031 \\
\mathrm{H} & 2.5507329306 & 2.0220744435 & 0.5300685785 \\
\mathrm{C} & 2.9384324085 & -0.8981978536 & -1.0572248506 \\
\mathrm{H} & 2.5539342306 & -0.5502598032 & -2.0181000034 \\
\mathrm{H} & 4.0377718714 & -0.9589687698 & -1.1205641049 \\
\mathrm{H} & 2.5453661992 & -1.9000567456 & -0.8728139147\end{array}$ & 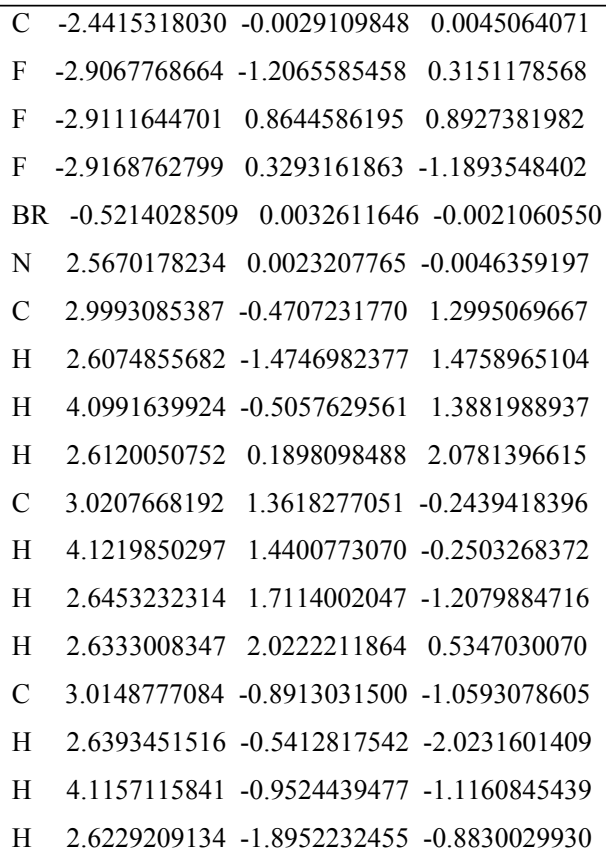 \\
\hline $\mathrm{Cl}_{3} \mathrm{C}-\mathrm{Br} \cdot \cdot \mathrm{NMe}_{3}$ & $\begin{array}{lrrr}\text { C } & 1.6003016911 & -0.0027300616 & 0.0044659668 \\
\text { CL } & 2.1956940557 & -0.6527435901 & 1.5470055972 \\
\text { CL } & 2.2013432042 & -1.0141361207 & -1.3268186110 \\
\text { CL } & 2.2045418775 & 1.6551148838 & -0.2028818299\end{array}$ & $\begin{array}{lccc}\text { C } & 1.8539520491 & -0.0029941841 & 0.0047829952 \\
\text { CL } & 2.4478139690 & -0.6099603466 & 1.5639733841 \\
\text { CL } & 2.4547692708 & -1.0498764906 & -1.2972264794 \\
\text { CL } & 2.4566454250 & 1.6478241459 & -0.2475427912\end{array}$ \\
\hline
\end{tabular}




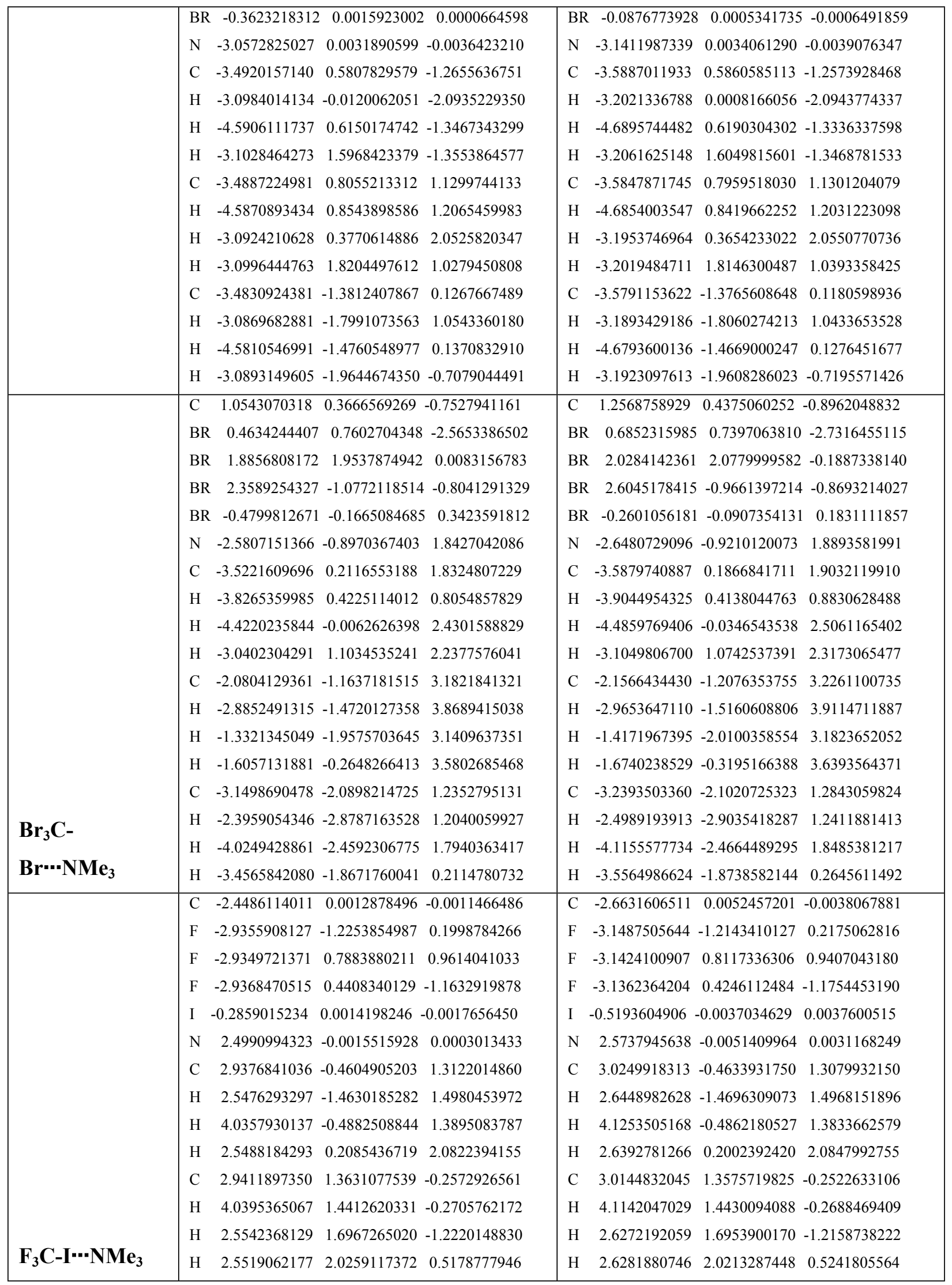




\begin{tabular}{|c|c|c|}
\hline & $\begin{array}{llll}\mathrm{C} & 2.9411691136 & -0.9078294547 & -1.0519141202 \\
\mathrm{H} & 2.5542205526 & -0.5672888529 & -2.0142071025 \\
\mathrm{H} & 4.0395597624 & -0.9603029894 & -1.1112461946 \\
\mathrm{H} & 2.5525399165 & -1.9095760848 & -0.8591028902\end{array}$ & $\begin{array}{llll}\mathrm{C} & 3.0217297046 & -0.9039779702 & -1.0493630428 \\
\mathrm{H} & 2.6332853392 & -0.5660123795 & -2.0124525124 \\
\mathrm{H} & 4.1218939577 & -0.9525603492 & -1.1146734770 \\
\mathrm{H} & 2.6420607268 & -1.9103646881 & -0.8606187575\end{array}$ \\
\hline $\mathrm{Cl}_{3} \mathrm{C}-\mathrm{I} \cdot \cdot \mathrm{NMe}_{3}$ & $\begin{array}{lccc}\mathrm{C} & -2.4005220868 & 0.0009810393 & -0.0016652427 \\
\mathrm{CL} & -3.0084090726 & 0.9500885660 & 1.3760650682 \\
\mathrm{CL} & -3.0063103251 & 0.7212092724 & -1.5126108236 \\
\mathrm{CL} & -3.0100716798 & -1.6661788046 & 0.1299671160 \\
\mathrm{I} & -0.1962640217 & -0.0016900169 & -0.0000114262 \\
\mathrm{~N} & 2.4717798564 & -0.0014697070 & 0.0014744882 \\
\mathrm{C} & 2.9110393047 & -0.4375527238 & 1.3231465038 \\
\mathrm{H} & 2.5206007335 & -1.4364196329 & 1.5249509209 \\
\mathrm{H} & 4.0084404535 & -0.4616391209 & 1.3961983219 \\
\mathrm{H} & 2.5206066843 & 0.2452127532 & 2.0797113232 \\
\mathrm{C} & 2.9117454149 & 1.3608758348 & -0.2820012617 \\
\mathrm{H} & 4.0091798651 & 1.4353756304 & -0.2986111033 \\
\mathrm{H} & 2.5205983971 & 1.6751332647 & -1.2511161542 \\
\mathrm{H} & 2.5223729036 & 2.0354298823 & 0.4823820560 \\
\mathrm{C} & 2.9112167254 & -0.9281718417 & -1.0369766793 \\
\mathrm{H} & 2.5203222136 & -0.6038026793 & -2.0028954485 \\
\mathrm{H} & 4.0086167289 & -0.9787409864 & -1.0948154300 \\
\mathrm{H} & 2.5212845350 & -1.9249254197 & -0.8239982788\end{array}$ & $\begin{array}{lccc}\text { C } & -2.6550703237 & -0.0004647233 & -0.0013241903 \\
\text { CL } & -3.2620660457 & 0.8346364497 & 1.4456179590 \\
\text { CL } & -3.2583035398 & 0.8355635591 & -1.4493585360 \\
\text { CL } & -3.2581328062 & -1.6723775727 & -0.0026936958 \\
\text { I } & -0.4844694505 & 0.0012399512 & 0.0013301964 \\
\text { N } & 2.5623115606 & -0.0001746241 & 0.0011509049 \\
\text { C } & 3.0074289207 & -0.4121080686 & 1.3237137040 \\
\mathrm{H} & 2.6195965955 & -1.4078067007 & 1.5490618323 \\
\mathrm{H} & 4.1072932501 & -0.4395565230 & 1.4022821879 \\
\mathrm{H} & 2.6243009549 & 0.2830100706 & 2.0737802173 \\
\mathrm{C} & 3.0116374825 & 1.3496546863 & -0.3039777579 \\
\mathrm{H} & 4.1117428875 & 1.4276419139 & -0.3209629706 \\
\mathrm{H} & 2.6283481854 & 1.6533639434 & -1.2804276306 \\
\mathrm{H} & 2.6271754606 & 2.0439641544 & 0.4461667617 \\
\mathrm{C} & 3.0065561174 & -0.9407889209 & -1.0161237594 \\
\mathrm{H} & 2.6210951680 & -0.6371034699 & -1.9917227766 \\
\mathrm{H} & 4.1063764217 & -0.9980402615 & -1.0774057452 \\
\mathrm{H} & 2.6204057912 & -1.9369385543 & -0.7899127511\end{array}$ \\
\hline $\mathrm{Br}_{3} \mathrm{C}-\mathrm{I} \cdot-\mathrm{NMe}_{3}$ & $\begin{array}{lccc}\mathrm{C} & -2.3872485687 & 0.0010326005 & -0.0013286492 \\
\text { BR } & -3.0504803881 & 1.1262532045 & 1.4454347113 \\
\text { BR } & -3.0496780811 & 0.6931519804 & -1.6988116569 \\
\text { BR } & -3.0540694742 & -1.8133767068 & 0.2485296232 \\
\mathrm{I} & -0.1830719889 & -0.0016759663 & -0.0004225951 \\
\mathrm{~N} & 2.4667343103 & -0.0017533441 & 0.0011341135 \\
\mathrm{C} & 2.9061905581 & -0.4530851474 & 1.3180961502 \\
\mathrm{H} & 2.5158614469 & -1.4542668303 & 1.5081286426 \\
\mathrm{H} & 4.0034691717 & -0.4772605585 & 1.3899199700 \\
\mathrm{H} & 2.5152690698 & 0.2206857205 & 2.0823358722 \\
\mathrm{C} & 2.9067410676 & 1.3643361391 & -0.2663078353 \\
\mathrm{H} & 4.0040688988 & 1.4378633194 & -0.2815936858 \\
\mathrm{H} & 2.5155340267 & 1.6896427400 & -1.2316683404 \\
\mathrm{H} & 2.5173363231 & 2.0295982352 & 0.5060888956 \\
\mathrm{C} & 2.9070951884 & -0.9162927710 & -1.0482188699 \\
\mathrm{H} & 2.5164448261 & -0.5804814146 & -2.0101969004 \\
\mathrm{H} & 4.0044448165 & -0.9651756135 & -1.1049666701 \\
\mathrm{H} & 2.5173687384 & -1.9154886824 & -0.8468116978\end{array}$ & $\begin{array}{lccc}\mathrm{C} & -2.6447525863 & 0.0009862215 & -0.0000873348 \\
\mathrm{BR} & -3.3088609690 & 0.9809804385 & 1.5459107459 \\
\mathrm{BR} & -3.3087497605 & 0.8492305939 & -1.6223094959 \\
\mathrm{BR} & -3.3064735758 & -1.8287368396 & 0.0757714371 \\
\mathrm{I} & -0.4787468745 & 0.0022058034 & 0.0001482149 \\
\mathrm{~N} & 2.5597834085 & -0.0008798669 & -0.0001745319 \\
\mathrm{C} & 3.0028600769 & -0.4180753474 & 1.3215454042 \\
\mathrm{H} & 2.6149929404 & -1.4148345096 & 1.5422440103 \\
\mathrm{H} & 4.1025637880 & -0.4457191582 & 1.4018655469 \\
\mathrm{H} & 2.6180695181 & 0.2738170680 & 2.0737324257 \\
\mathrm{C} & 3.0087981321 & 1.3505502190 & -0.2989513238 \\
\mathrm{H} & 4.1088470123 & 1.4293991072 & -0.3133656982 \\
\mathrm{H} & 2.6266025561 & 1.6579237971 & -1.2746680240 \\
\mathrm{H} & 2.6223613054 & 2.0414249669 & 0.4533874019 \\
\mathrm{C} & 3.0061745996 & -0.9371721555 & -1.0205940126 \\
\mathrm{H} & 2.6221702517 & -0.6294879584 & -1.9955272364 \\
\mathrm{H} & 4.1061111235 & -0.9934361769 & -1.0803786630 \\
\mathrm{H} & 2.6202589951 & -1.9344692983 & -0.7992077886\end{array}$ \\
\hline
\end{tabular}

